\title{
The Comparison of 'Rewriting' of Howard Goldblatt \& Andre Lefevere's Translation Thoughts
}

\author{
Chunhua Shen \\ School of Foreign Languages, Wuhan Polytechnic University, Wuhan 430038, China.
}

Chunhua shen@163.com

Keywords:Goldblatt; Lefevere; rewriting theory; ideology; poetics; patronage; comparison

\begin{abstract}
This paper illustrates firstly the importance of the study of rewriting studies, and then respectively takes a closer look at the basic content of Lefevere's rewriting thoughts and Goldblatt's rewriting thoughts and then comparing their different views on rewriting thoughts. In the end, the paper gives a profound reflection on the development of this theory and its current situation while discussing the limitation of rewriting theory.
\end{abstract}

\section{Introduction}

Translation is an activity with a long history. Rewriting theory proposed by Lefevere reviews translation activities from the perspective of culture, provides a new perspective for translation studies, and also provides translation study with a new way, so as to expand the field of translation studies. This theory is out of the barrier of texts, liberating the translation studies from the shackles of pure language transformation to focus on the macroscopic cultural factors affecting the whole literary system, which promotes the translation studies paradigm transformation, expands the research field of translation, thus gives a stronger vitality to translation study and promotes the development of translation. But the theory is also deficient, such as the fuzzy definition, ignore value judgment, give little attention to the recognition of translator's subjectivity and so on. So it is necessary to criticize and reflect on Lefevere's rewriting theory. Through debates, criticism, objective and fair evaluation of its academic achievements as far as possible, so the development of translation studies has more vitality.

\section{The Three Important Factors of Lefevere's Rewriting Theory.}

In 1990, the concept "rewriting" was firstly put forward by Andre Lefevere in his monograph "Translation, Rewriting and Manipulation of the Literary Reputation. Putting translation in the context of culture, Lefevere pointed out that translation is not only a language change between text, but also the rewriting of the original works based on politics, culture, consciousness level in the target language society. In his book, he put forward the rewriting theory composed by the "ideology", "poetics" and "patron". He pointed out that in different times, rewriting was mainly limited by ideology, poetics. With the consideration of social ideology and poetics, the translator rewrites the original one for the sake of making it conform to the mainstream consciousness and poetic form and letting the translation understood by much more readers. Ideology, understanding and cognition of things, is a kind of sense. And it concludes concept, ideas, concepts, values and other factors combined. People's Ideology is influenced by thinking ability, environment, information, education, publicity, value orientation and other factors. Thus different ideology has different understanding and cognition to the same thing.

There are a lot of differences between Chinese and western religion. Specifically, Christianism takes the mainstream in western countries, while in China, the dominated religions are the native Buddhism and Taoism. So in Chinese literature, the gods from Buddhism, Taoism appears frequent, and it is difficult to find equivalence in western ideology.Translation is influenced by national mainstream poetics and the translator's aesthetic temperament. For example, in translating one sentence in Kong Yiji, William A and Yang Xianyi give different translated version. (William A) ..."The purloining 
of volumes, good sir, cannot be controlled as theif. The purloining of volume is , after all, something that falls well within the purview of the scholarly life. How can it be considered mere theft?" Tacked onto that was a whole string of words that were difficult to understand, things like the gentleman doth stand firm in his poverty, and verily this and forsooth that. (Yang Xianyi ) ..."Taking a book can't be counted as stealing." Then followed such quotation from the classics, like"A gentlemen keeps his integrity even in poverty," and a jumble of archaic expression...By comparing the two versions of translation, Yang Xianyi's version is featured with concise and intelligible while William A's version highlights the characteristics of Kong Yiji. Because of their different views of poetics, they has taken a different translation strategies about punctuation. Yang Xianyi's version preserves the ellipsis of the original version, which fully embodies Kong Yiji's nervous mood at that time. As for the translation of the reference of ancient prose, such as "the gentleman", "instant poor" and "zheye", William A uses many old arcane English words like "forsooth", which straightly gives readers a sense that Kong Yiji is very pedantic, and vividly displays a poor intellectual poisoned by the feudal education in front of the readers. Compared with William, Yang Xianyi adopts modern language, translating all "zheye" into "archaisms". Thus, his translation lacks competition. Lefevere holds the view that patrons also influence the effect of translation. The patrons he mentions mainly include the external forces such as investors or institutions, which mainly refers to press involvement. The demand of the market is the power source of press. When publishing a work, the press is bound to make forecast to its sales, for the press only makes great efforts to publish the works hoped by mass audience. Mo Yan, a Chinese writer, becomes a budding star in the world for his earliest work Red Sorghum. After enjoying the magic of the combination of Hallucinatory realism and folk tales, people become keener to get access to this Chinese contemporary novelists and his works, which gives great impetus to press to publish Mo Yan's other works. On the other hand, when translating Big Breasts and Wide Hips, the press requests Howard Goldblatt to omit the tedious plots and the cultural revolution in order to comply with the social norms and cater to readers' aesthetic psychology. As a translator, Howard Goldblatt had to obey the manipulation of "external forces" like patrons. The three factors of Lefevere's rewriting theory play an very import role in translation. In different times, different ideology may influence the choice of translation targets, the text which needs to be translated and the translation strategies. Even in the same time, different people have different ideology, which is bound to bring effect to the translation activities. As for poetics, it decides the translation's social role. And too much emphasis on meaning while neglecting poetics will reduce the beauty of the original work. Lastly, patrons are the most important factor. They manipulate the whole process of translation. In another words, translators in all generations are engaged in translation activities with the permission of politic power. Inevitably, translation activities are always controlled by the three factors.

\section{Goldblatt's rewriting thoughts when translating.}

Howard Goldblatt, a famous American translator, was born in 1939. During the 1960s, he learned Chinese in Taiwan while serving in the military. Later he graduated from Indiana university and got a doctor degree in Chinese literature. And now he is in the highest position in the English world as a translator of Chinese literature. Rigorous and devoted, he polishes Chinese literature with the color of contemporary British and American literature. Up to now, Howard Goldblatt's translation lists includes Xiao Hong, Chen Jo-hsi, Bai Xianyong, Leon, Zhang Jie, Yang Jiang, Feng Jincai, che, Jia Pingwa, Li Rui, Liu Heng, Su tong, old ghost, Wang Shuo, Mo Yan, rainbows, loy, chu Tien-wen or more than two dozen famous writers. There is no doubt that Howard Goldblatt plays an important role to Chinese literature "going out" .

Reader-oriented. All Howard Goldblatt's translations are enclosed by one or several compensation method, such as translator prefaces, the main characters lineage tables, charts, pronunciation, annotations etc. so as to help readers understand the background and the clues of the story. Most of Goldblatt's translations are attached with translator prefaces, which explain the relative information and omission of the original context. For example, in the preface of The Republic of Wine, he points out that Mo Yan uses a lot of puns. Besides, proses of all sorts of styles, allusions, politics, literature, 
elegance and vulgar coexist. What's more, there are many Shandong dialect. He holds the view that now that readers can not fully understand the forms of literature, there is no need to explain them in detail. And he confesses to the readers that he will try his best to be faithful to Mo Yan's works, but he failed to do so. All the hope that the revised translation can make up the deficiency. As for main character pedigree table, we can take Big Breast and Wide Hip as an example. According to the table, information about the character mother are as follows: her maiden name is snow; since childhood, she lost her parents and was brought by his uncle; Then she converted to Christianism. Introductions about seven daughters are even more detailed. He specifically introduces the sons-in-law's professions, the names of their kids, daughter born child relationship is very clear. Howard Goldblatt in Wolf Totem translators attach a map of China, after introduction of can make readers through the map to better grasp the background of the story. Translations with pronunciation annotation are not universal. In the Life and Death, Howard Goldblatt encloses pronunciation annotation with the main character table so as to tell readers that the pronunciation way of the majority of Chinese pinyin is consistent with English words, except several pinyin such as "e" "he" "ian" "le" "qi" "x" "zh”. This do help readers understand the standard of Chinese pronunciation.

Combination of translators' subjectivity and principle of Simplification. When translating, Goldblatt takes cultural default, restructures events, deletes the unnecessary narrations and descriptions, integrates short sentences to simplify the original text, which increases the readability of the translation. Principle of Simplification makes Goldblatt translations more consistent with the target language readers' reading expectations in terms of narrative structure. According to English readers' habit, Goldblatt gives full play to translator's subjectivity during the translation practice. He writes to Mo Yan, suggesting him to modify the end of Song of Garlic Stalks in Tiantang County. Later, the English version comes into its publishing smoothly. For the widely acceptance of the English version, the other language versions also adopt the end of Goldblatt's English version. Eli Zohar's multiple system theory can fully explain Howard Goldblatt's translation choices. In the 1990s, Chinese literature translation was at the edge of the multiple system theory. At the same time, western literature tends to leave out preface. Thus Goldblatt complys with the culture of the target language, deleting the preface of the "Wolf totem" . All in all, what Howard Goldblatt pursuit is to find the best model of foreign text and the acceptability of the translation by the target language readers.

Translation is rewriting. According to my experience, most writers should at least tolerate those who are given the task to rewrite their works with other languages, because the nature of translation is rewriting.(Goldblatt, 2002) Goldblatt had ever taken "god" as an example, which often appeared in the Dust Settles Down. From the literal meaning, "Heavens" is the closest equivalent word to "god". However, it is not suitable to translate all "god" into "Heavens". After trying several options, he and his wife discussed to bring in other languages, letting different people say different "my god", such as "Aicaramba", "Achdulieber", "Mamamia", "Oygevalf", "Merde". These, he thought, were more vivid than English interjections. Therefore, translation has never been a copy, but to rewrite in a different language. It is an improvement of the original text, even a perfect version. Another example is "Anybody who doesn't drink doesn't deserve to be called a man...". This is one sentence of Li Bai's poem, which means that people should fully enjoy life whenever possible. This sentence is often used to persuade others to enjoy immediate pleasures. In this novel, it is used to urge others to drink. Instead of directly expressing the meaning of poetry, the function and the meaning is same.

\section{The limitation of the rewriting theory.}

Rewriting theory pays little attention to the micro-level translation practices, specifically, it attaches little importance to the micro-level text study and the study among texts. Factors hiding behind texts such as patrons, poetics, ideology are paid much attention by this theory. It guides people to turn the vision of translation studies to cultural fields. Rewriting theory expands the scope of translation studies, and provides a new criteria for translation activities, but what it discusses is the outside factors of translation studies rather than the inner factors. Translation refers to find the closest and most natural equivalent in the target language in terms of semantic and text. Therefore, we cannot ignore bilingual study and the study of inner factors. Functional translation theory emphasizes that translation is a special kind of communication form, which cannot leave the text study, and text 
design has three kinds of forms: the original texts, scheme text and target language text. As for original texts, the most important thing is to catch the rhetoric function created by the author and understand the correct meaning, which is the key to produce ideal schema text. And correctly mastering the cognitive schema of the original text is the basis of correct understanding of the original rhetorical functions. Ideal scheme texts come from the original scheme and the correct understanding of the author's rhetorical intent. On the basis of schema texts, we should consider how to translate texts, the equivalence of rhetorical functions and the target readers. In short, only through in-depth research can such problems be solved.

Rewriting theory overstates the role of the mainstream ideology while ignoring the effect of non-mainstream ideology, especially the response of the translator. During the May fourth movement, China is a semi-feudal and semi-colonial country. The Chinese people were oppressed and exploited by the imperialist and the feudal forces. Some advanced intellectuals fumbled the truth of life, hoping to find the way to save the nation from subjugation, and translation is an important means of cultural transmission. Thus in the May fourth period, the Russian literature, literature in damaged nations, and realism literature and romantic literature in France, Germany, Britain, northern Europe were translated. But for the same mainstream ideology, different translators have different reactions. During this period, Bing Xin, Lu Xun and other people have a "weak" preference while Hu Shi, Qu Qiu-bai have "strong” plot. Specifically, Bing Xin and Lu Xun have the preference to translate literature form the third world countries such as India, Belgium, the Czech republic and other countries; But Hu Shi and Qu Qiu-bai choose to translate literature from western developed countries. Therefore, in addition to the content of the rewriting theory, factor manipulating translation activities also includes translators' response, namely the translator's preference of the texts.

As long as Rewriting theory adopt the description method of description translatology, it will do benefit to summarize translation experience, find the rule guiding translation practice so as to objectively reflect the value of translation. But as a scientific research method, it's very difficult to achieve pure description without the consideration of value judgment. On the other hand, looking from the overall tendency of the studies on the translation, we can find that in the 1950s, the western translation theories still gave priority to stipulation, while in China, Chinese traditional translation theory develops from fidelity to the original, faith to spirituality, sublimation, these thoughts also have a prescriptive method which regard the original language as the center. Thus we should evaluate its limitations and inevitability objectively. Rewriting theory puts too much emphasis on the description of the objection, lack of value judgment to different rewriting forms, and ignore the negative effects of uncertain factors. seeing from above, its irrationality is bound to lead to chaos to rewriting itself.

\section{Summary}

Translation, as a kind of cross-language and cross-cultural communicative activity, is not only a mechanical conversion between source language and target language, but a flexible conversion under the joint influence of all factors like social and cultural factors. Rewriting theory, as a translation theory, plays a vital role in translation activities. Although there is some difference between Lefevere and Goldblatt's rewriting thoughts, their basic goal is same. That is to give impetus to the translation career. Up to now, the limitation about rewriting theory exists, but with these two people's great guidance, we believe that this theory will be more perfect and constructive to translation activities.

\section{References}

[1]Aitchison, (1996) The Seeds of Speech: Language Origin and Evolution. Cambridge:Cambridge University Press.

[2]Belloc, H. (1931) On Translation. Oxford: Oxford University Press.-(1931a) “On Translation” Bookman 74:32-9, 179-85. 
[3]Bergonzi, B. (1990) Exploding English: Criticism, Theory and Culture. Oxford: Clarendon Press. 